2009-01-09

\title{
Generation of internal waves by a supercritical stratified plume
}

\author{
Stashchuk, Nataliya
}

http://hdl.handle.net/10026.1/3851

10.1029/2008jc004851

Journal of Geophysical Research

American Geophysical Union (AGU)

All content in PEARL is protected by copyright law. Author manuscripts are made available in accordance with publisher policies. Please cite only the published version using the details provided on the item record or document. In the absence of an open licence (e.g. Creative Commons), permissions for further reuse of content should be sought from the publisher or author. 


\title{
Generation of internal waves by a supercritical stratified plume
}

\author{
Nataliya Stashchuk ${ }^{1}$ and Vasiliy Vlasenko ${ }^{1}$ \\ Received 6 April 2008; revised 7 October 2008; accepted 24 October 2008; published 9 January 2009.
}

[1] The generation of internal waves by a propagating river plume is studied in the framework of a fully nonlinear nonhydrostatic numerical model. The vertical fluid stratification, parameters of tide, river discharge, and the bottom topography were taken close to those observed near the Columbia River mouth. It was found that in the beginning of the ebb tidal phase the river water intruding into the sea behaves as a surface jet stream. It collides with the stagnant shelf waters and sinks down in the area of the outer plume boundary, forming a head of the gravity current. In supercritical conditions which are normally realized at the first stage of the ebb tidal phase, internal waves are arrested in the head of the gravity current because their phase speed is smaller than the velocity of the plume. They are released and radiate from the plume when the speed of the decelerating front becomes smaller than the internal wave phase speed. This mechanism of the wave generation is sensitive to the stratification of the ambient shelf waters. It was found that dramatic decay of the buoyancy frequency profile from the surface to the bottom provides the most favorable conditions for the efficient disintegration of the head of the gravity current into a packet of internal waves and their fast separation from the plume. In the case when the fluid stratification on the shelf is close to monotonous, the disintegration of the head of the gravity current into a packet of solitary internal waves is not expected.

Citation: Stashchuk, N., and V. Vlasenko (2009), Generation of internal waves by a supercritical stratified plume, J. Geophys. Res., 114, C01004, doi:10.1029/2008JC004851.

\section{Introduction}

[2] The nonlinear internal waves (IWs) are widely acknowledged as being a small-scale process with global and climatic significance. They play a fundamental role in water mixing and, as a result, in the setting the global oceanic density structure. It is well established and proved nowadays fact that one of the most powerful and regular sources of internal waves is the conversion of barotropic tidal energy into baroclinic component occurring over large-scale bottom features, that is, continental slopes, oceanic ridges and banks (see, for instance, Garrett and Kunze [2007] and references herein).

[3] As distinct from the tidal energy conversion, there are, however, some other important sources of oceanic internal waves: shear instability, atmospheric impact, local initial disturbances of buoyancy fluxes and stratified currents [Thorpe, 2005]. A relatively new mechanism of IWs generation has been formulated recently by Nash and Moum [2005] who reported the observation of packets of IWs in the area of the Columbia River mouth and assumed that they were generated by the river plume in the coarse of its transition from supercritical to subcritical regime of evolu-

\footnotetext{
${ }^{1}$ School of Earth, Ocean and Environmental Sciences, University of Plymouth, Drake Circus, Plymouth, UK.

Copyright 2009 by the American Geophysical Union. 0148-0227/09/2008JC004851\$09.00
}

tion. These packets are regularly observed from space by synthetic aperture radars (SAR) [Fu and Holt, 1982]. Note, however, that one should distinguish two type of internal wave packets appearing in SAR images near the Columbia River plume. The first type is the "classical" system of IWs generated by the barotropic tide over the shelf break according to the mechanism of tidal energy conversion mentioned above. Such waves propagate shoreward; they are presented in Figure 1 by the fragments with symbol I (for details, see Moum et al. [2003]). The second group of waves has a circular fronts (find fragments marked by II in Figure 1); they propagate seaward and have completely different origin. It is assumed that these circular waves are generated by the Columbia River plume.

[4] The Columbia River is the largest river on the west coast of North America with an average discharge of $\sim 260 \mathrm{~km}^{3}$ year ${ }^{-1}$. The mouth of the river is located at approximately $\sim 46^{\circ} \mathrm{N}$, entering the Pacific Ocean in the area of a relatively straight meridionally oriented coastline with a narrow shelf. Because of a strong semidiurnal tidal activity, the river water intrudes into the sea twice a day with ebb currents forming a thin surface low-salinity lens which extends more than $10 \mathrm{~km}$ from the mouth. Penetrating into surrounding waters the plume behaves as a surface gravity current with the head developed at the boundary between the plume and the sea.

[5] Weakly nonlinear theory predicts that one of the most probable scenarios of evolution of any localized initial 


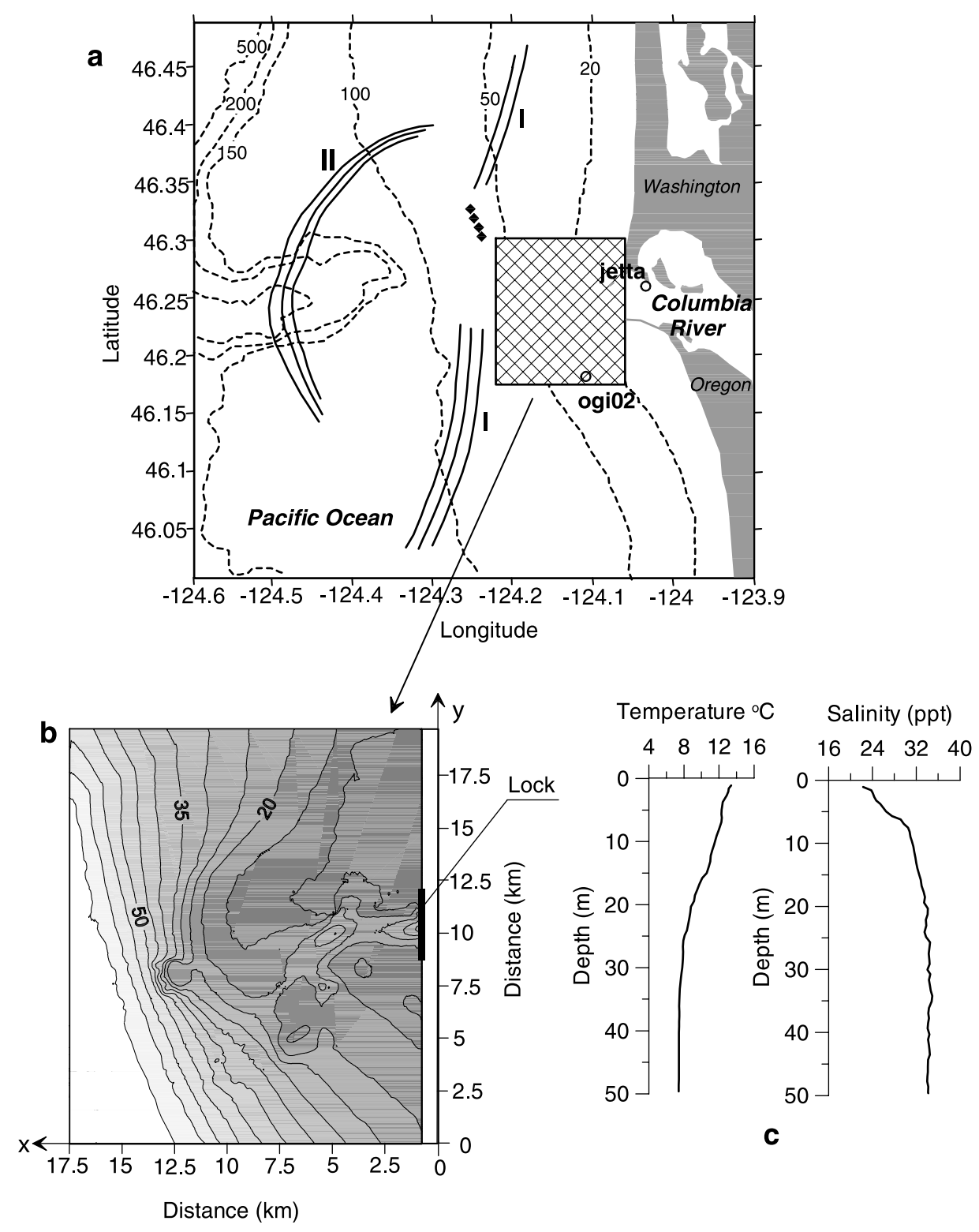

Figure 1. (a) The fragment of the Pacific Ocean showing the surface manifestations of IWs (marked as I and II) acquired by SAR. The black diamonds spot the position of measurements [Nash and Moum, 2005]. Two open circles show the location of CTD stations: "Jetta" and OGI02. The shaded rectangle designates the model domain. (b) Zoom of the model domain. (c) The profiles of the background temperature and salinity on the shelf.

density disturbance is its disintegration into a system of solitary waves [Whitham, 1974]. The number and the intensity of radiated waves depends on spatial characteristics of the initial perturbation. Applying this idea to the Columbia River plume one can assume that in the beginning of the plume development its leading edge contains such a system of internal waves traveling with the plume. On the basis of their observations Nash and Moum [2005] assumed that the plume propagates faster than these waves at the first stage of the ebb phase. It was hypothesized that IWs are released and detach from the plume when its speed, $U_{p}$, becomes smaller than the phase speed of IWs, $V$. This transition from supercritical regime with $U_{p}>V$ to the subcritical one when $U_{p}<V$ is possible because both velocities, $U_{p}$ and $V$, vary in space and time. It is important also that in a very general case the IWs generated by the plume are nonlinear, which is why the velocity $V$ should be treated as a nonlinear phase speed (see section 3 ).

[6] There is, however, an alternative possible explanation of the waves generation by the moving plume. It is based on the resonant theory predicting the wave excitement by a moving pressure area [Akylas, 1984], or by a topography obstruction in a stratified flow [Grimshaw and Smyth, 1986]. In both cases the upstream propagating nonlinear internal waves appear as a formal solution of a forced Korteweg de-Vries (fKd-V) equation without disintegration of an initial disturbance. As a confirmation of this mechanism, the gravity current can be considered as a solid 

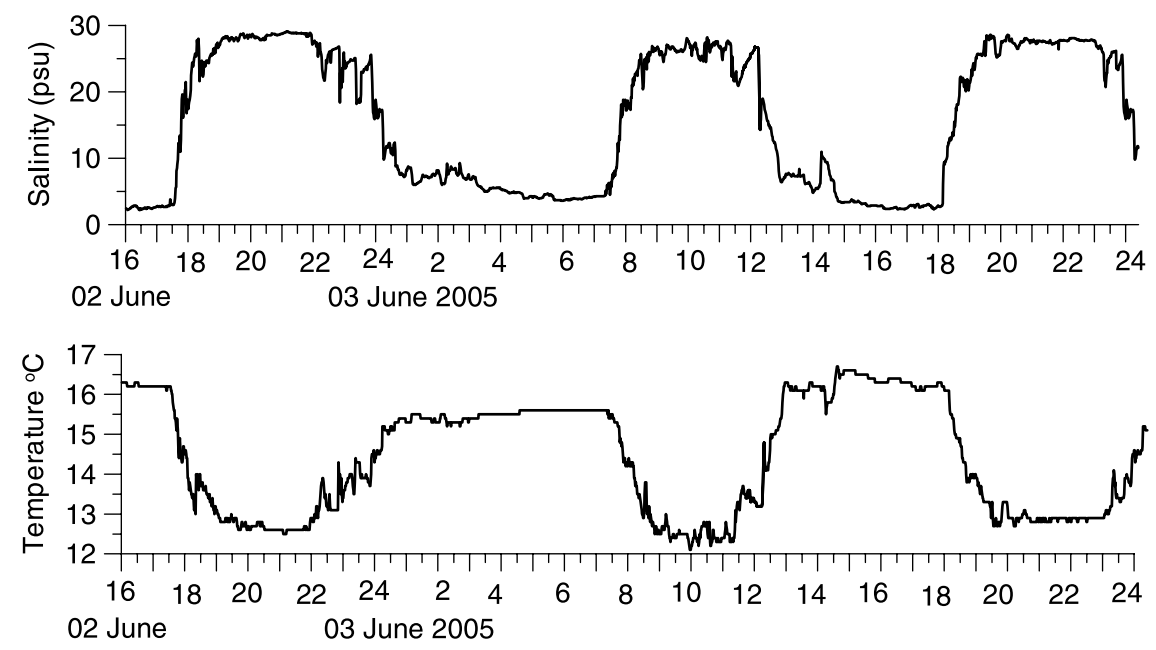

Figure 2. Example of CTD measurements in the river mouth at depth of $6.4 \mathrm{~m}$ (station Jetta).

"topographic" object propagating in surface layer (some kind of piston) at the first stage of fast plume spreading. Formally, all conclusions of the resonant theory can be applied to this object moving with speed $U_{p}$ and generating internal waves in front of it.

[7] To be precise, the resonance theory of a stratified flow over topography predicts the generation of internal waves propagating both upstream and downstream [Grimshaw and Smyth, 1986]. The most interesting effect develops in the case when the difference between the flow speed, $U_{p}$, and the phase velocity of IWs of the mode 1 internal waves, $V$, is small. In the beginning of the motion when the amplitude of generated wave is not large enough, the upstream propagating wave is arrested by incoming flow. The wave thus remains in the forcing region for a while and grows in amplitude (formation the head of the gravity current). The growth continues until the wave amplitude reaches a sufficiently large value to move upstream because of the nonlinear dispersion and escape from the "topography". According to the resonant theory the speed of the radiated wave can be equal, slightly greater or smaller than the flow speed. In the limit of a steady state flow, this topographic resonance results in the generation of an infinite train of periodically spaced solitary waves of uniform amplitude and fixed wave period. In the case of a decelerating flow the resonant theory predicts a generation of a rank-ordered packet of the solitary waves propagating upstream [Wang and Redekopp, 2001].

[8] The situation with a propagating freshwater plume is more complicated than the above scheme presents because the plume cannot be considered as a "solid" object. In fact, the plume lens is getting thinner in the course of propagation because of the radial spreading. In addition, a head of the gravity current really produces an internal bore which separates from the plume and disintegrates into a packet of internal waves according to the mechanism discussed by Whitham [1974]. In summary one can assume that both mechanisms contribute to the wave generation, although, some theoretical study would be helpful to clarify the conditions of the waves formation.

[9] Note that a forecasting estuarine numerical model, ELCIRIC, targeted on a prediction of water level, velocity, temperature and salinity fields in the area of the Columbia River plume [Zhang et al., 2004] is not able to reproduce a generation of short IWs by the plume because of the limitation imposed by the hydrostatic approximation. For investigation of the generation of IWs by a decelerating plume a nonhydrostatic model would be more appropriate. The Massachusetts Institute of Technology general circulation model [Marshall et al., 1997] is used in the present study in order to determine the major factors controlling the wave generation by the river plume and to identify the range of the input parameters making the generation process possible. The paper is arranged as follows: the model and its initialization are described in section 2; section 3 contains the results of the Basic Case Run (BCR), whereas the sensitivity of the model results to various input parameters is presented in section 4; influence of ambient stratification is investigated in section 5; discussion and summary of the present study are formulated in section 6 .

\section{Model Description}

[10] The dynamics of the Columbia River plume is studied within the domain shown in Figure 1. The detailed bottom topography is presented in Figure 1b. Consider the Cartesian system of coordinates, arranged in such a way that the plane $O x y$ is situated at the undisturbed free surface with the $O z$ axis directed vertically upward. Two other axis, $O x$ and $O y$, are arranged perpendicular and along the shoreline, respectively. The basin (a sector $L_{x} \times$ $L_{y}=16.775 \times 19.7 \mathrm{~km}^{2}$ ) is filled with stratified fluid whose salinity and temperature profiles are taken close to those measured outside the estuary (Figure 1c).

[11] An important stage of the model initialization is the correct parameterization of the freshwater discharge from the estuary. Figure 2 represents the time series of the salinity and the temperature at 6.4-meter depth recorded at station Jetta in the river mouth (note that data in Figure 2, as well as in Figures 3 and 8d were taken from the Web site: http:// www.ccalmr.ogi.edu/CORIE/network/jetta/). It is clear from Figure 2 that the temperature and the salinity in the estuary alter with tidal periodicity. Another conclusion comes from the analysis of their range: strong variability of the temper- 
24 June 2004
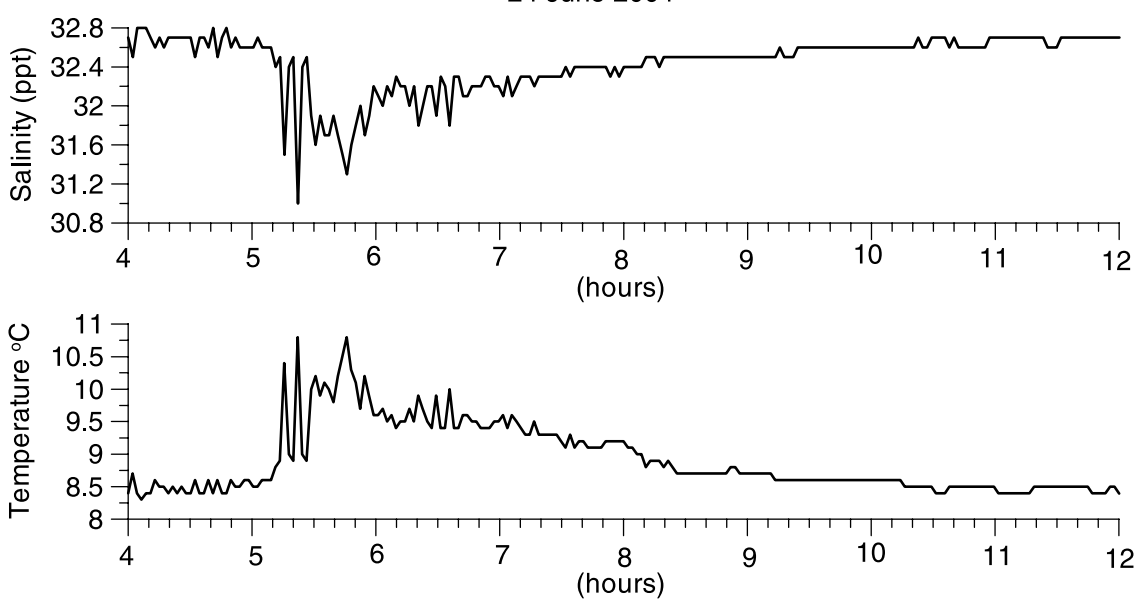

Figure 3. Time series of temperature and salinity recorded by station OGI02 at depth of $10 \mathrm{~m}$.

ature and the salinity, that is, $\Delta T>4^{\circ} \mathrm{C}$ and $\Delta S>25 \mathrm{ppt}$ means that water properties at Jetta vary from almost fresh river water during the ebb phase to pretty saline seawater in the flood phase. Combining these two facts one can conclude that the barotropic tidal flow is the major driving force controlling a semidiurnal periodicity of the freshwater discharge. Saline seawater is pumped into the estuary during the flood tidal phase, whereas the ebb phase is characterized by a strong outflow of almost fresh river water in opposite direction. It is important that the transition from the fresh to saline water regime in the mouth occurs pretty fast as if there is a lock in the mouth which operates with a semidiurnal periodicity. Thus a "lock-exchange" approach in the modeling of sea-river interaction is well justified. An additional sector of $1 \times 19.7 \mathrm{~km}^{2}$ attached to the model domain to the right of the lock is shown in Figure 1b by white color. This "ad hoc" extra basin is volumetrically equal to the Columbia River estuary. Two basins (the inner and the outer) are connected through a narrow gap initially closed by a lock. The width of the gap was taken equal to $3.5 \mathrm{~km}$, which is close to the actual mouth width.

[12] The water inside the estuary is taken homogeneous at $t=0$ with the salinity $S_{e}=8 \mathrm{ppt}$ and the temperature $T_{e}=$ $20^{\circ} \mathrm{C}$. When the lock is released, the river water starts to move from the estuary into the sea as a surface gravity current. The latter is produced by the action of three major forcing: the ebb tidal flow, river discharge and the horizontal pressure gradient caused by the density difference between the sea and the estuary.

[13] It is ought to note that the measurements of Nash and Moum [2005] were conducted close to the model domain (find black diamonds in Figure 1a) whereas the position of IWs packet recorded by the RADARSAT-1 satellite (fragment II in Figure 1a) is located much farther. Another evidence of internal waves generated by the plume is shown in Figure 3 which represents the time series of the salinity and the temperature recorded by station OGI02 at 10-meter depth. These profiles can be treated as a solibore with two well-defined leading waves detached from the head of the gravity current. Figure 3 illustrates the fact that the waves can be generated by the plume in the close proximity to the mouth. To provide the conditions for the generation of IWs within the model domain, the relatively weak river discharge, that is, $Q=5000 \mathrm{~m}^{3} \mathrm{~s}^{-1}$ (which actually varies from 4000 to $18,000 \mathrm{~m}^{3} \mathrm{~s}^{-1}$ ) and a moderate value of tidal ebb velocity, $U_{\text {tide }}=0.5 \mathrm{~ms}^{-1}$ [Hickey and Banas, 2003], have been chosen for the model initialization.

[14] Another important point for the modeling is the correct choice of the grid. According to the observations [Nash and Moum, 2005; Pan et al., 2007], the scale of the recorded IWs is of about $200 \mathrm{~m}$. So, the horizontal step $\Delta x=\Delta y=25 \mathrm{~m}$ looks small enough to achieve necessary resolution of IWs. However, some runs were performed with twice smaller spatial step, $\Delta x=\Delta y=12.5 \mathrm{~m}$, to study sensitivity of the model to the spatial resolution (see section 4). In vertical direction the plume presents a thin lens with the mean depth of about $10 \mathrm{~m}$ [Orton and Jay, 2005], where fine vertical resolution should be provided. The following series of vertical step $\Delta z=1,1,1,1,1,1$, $1.5,2,2.53,5,10,10 \mathrm{~m}$ (from the top to the bottom) was taken in the model.

[15] Assuming strong vertical water mixing, the Richardson number-dependent parameterization [Pacanowski and Philander, 1981] is chosen:

$$
\nu=\frac{\nu_{0}}{(1+\alpha \mathrm{Ri})^{n}}+\nu_{b}, \quad \kappa=\frac{\nu}{(1+\alpha \mathrm{Ri})}+\kappa_{b},
$$

where $v$ and $\kappa$ are the coefficient of the viscosity and diffusivity, respectively, $\mathrm{Ri}$ is the Richardson number, $\mathrm{Ri}=$ $N^{2}(z) /\left(u_{z}^{2}+v_{z}^{2}\right)$ and $N^{2}=(-g / \rho \partial \rho / \partial z)^{1 / 2}$ is the buoyancy frequency, $g$ is the acceleration due to gravity, $\rho$ is the density. Here $v_{b}=10^{-3} \mathrm{~m}^{2} \mathrm{~s}^{-1}$ and $\kappa_{b}=10^{-5} \mathrm{~m}^{2} \mathrm{~s}^{-1}$ are the background dissipation parameters, $v_{0}=1.510^{-2} \mathrm{~m}^{2} \mathrm{~s}^{-1}$, $\alpha=5$ and $n=1$ are the adjustable parameters. Such a parameterization for the vertical turbulent viscosity $v$ and diffusivity $\kappa$ increases their values in the areas where the Richardson number is small. Horizontal diffusivity and viscosity were set at the level of $0.5 \mathrm{~m}^{2} \mathrm{~s}^{-1}$.

[16] The Orlanski open boundary conditions were used at the western, northern and southern boundaries of the outer basin [Marshall et al., 1997]. The model estuary (the white-color rectangular shape to the right of the lock, see Figure 1b) was closed at its northern and southern bound- 
aries. At the eastern boundary, $x=0$, the temperature and the salinity are set $T_{e}=20^{\circ} \mathrm{C}$ and $S_{e}=8 \mathrm{ppt}$, respectively (see Figure 2) and the horizontal velocity is found from the formula $u=-Q /\left(H L_{y}\right)-U_{\text {tide }} * \sin \left(2 \pi * t / T_{\text {tide }}\right)$, which sets a superposition of the river discharge (the first term) and periodical tidal current (the second term). Here $Q$ is the volume of river discharge, $H$ is the local water depth and $T_{\text {tide }}$ is the tidal period.

\section{Model Results}

[17] In investigation of the Columbia River plume dynamics two quite different processes should be traced at the same time. The first one is the development of the plume which is mostly a surface phenomenon. Its position and spatial structure can be visualized using sharp surface salinity gradients. Hence the best way to illustrate the plume position is to analyze the salinity field at the free surface. Unfortunately, the generated IWs (the second object of our analysis) do not produce any salinity signal at the free surface. They can be recorded at depth by remarkable displacement of isopycnals, instead. Note, that in the present study the plume and generated IWs should be traced together. The situation can be rectified and the spatial structure of IWs can be recovered at the free surface if one takes into account the ability of IWs to produce zones of strong convergence and divergence there. This property of IWs is used for their satellite tracking, for instance. According to Alpers [1985], there is a biunique relationship between the brightness of a signal appeared in SAR images and the gradient of horizontal velocity at the surface, $G=$ $\left[(\partial u / \partial x)^{2}+(\partial v / \partial y)^{2}\right]^{1 / 2}$. It was found in practice [see Alpers, 1985] that internal wave signal is clearly identified by SAR if the gradient $G$ is larger than $10^{-3} \mathrm{~s}^{-1}$. We take this value, that is, $G=10^{-3} \mathrm{~s}^{-1}$, as a practical threshold for identification of IWs.

[18] Thus the investigation of the generation of IWs by a decelerating plume can be done by a joint analysis of the salinity and the velocity gradients at the free surface combined in one figure. An example of such an approach is shown in Figure 4 where the fields $S(x, y, 0, t)$ and $G(x, y$, $0, t)>10^{-3} \mathrm{~s}^{-1}$ are overlaid. In the Basic Case Run (BCR) presented in Figure 4 all three driving forces were activated: (1) the density difference $\Delta \rho$ between the estuary with $\rho_{e}=$ $1001.92 \mathrm{~kg} \mathrm{~m}^{-3}$ and the seawaters with $\rho_{s}(0)=1013 \mathrm{~kg}$ $\mathrm{m}^{-3}$ at the surface (and $\rho_{s}(H)=1026 \mathrm{~kg} \mathrm{~m}^{-3}$ at the bottom), (2) the river discharge with $Q=5000 \mathrm{~m}^{3} \mathrm{~s}^{-1}$, and (3) the ebb tidal flow with maximum velocity $U_{\text {tide }}=0.5 \mathrm{~m} \mathrm{~s}^{-1}$.

[19] Figure 4 shows that the spatial structure of the salinity field within the plume is highly intermittent. Just after the beginning of the motion (first hour of the experiment), the plume boundary is nearly symmetrical, and the salinity field inside the plume is mostly uniform. The symmetry and homogeneity are violated at the latest stages of the evolution when one can clearly identify the jet stream in the middle of the plume which supplies the fresh water from the mouth. The water around the jet is more saline. The jet oscillates in space and time revealing quite unstable behavior. Its axis slightly turns to the left whereas the basic part of the plume is shifted to the right according to the Coriolis effect (see Figure 4). Similar behavior of the plume was discovered by Horner-Devine [2008] on basis of the observational data. The reason of unexpected jet deviation is conditioned by the shape of the bottom topography in the area: in fact, there is a canyon near the mouth located just at the exit from the estuary. The areas adjacent to the canyon are extremely shallow (less than 10-m depth) and provide a natural barrier for the jet. It seems that the canyon flanks prevent the jet from its turning to the right. As a result, the freshwater follows a general route of the canyon. This issue is discussed in section 4 in more detail.

[20] Let us focus now on the spatial structure of the generated IWs. They are presented in Figure 4 by the thick lines where $G>10^{-3} \mathrm{~s}^{-1}$. It is seen that during the first two hours of the experiment the position of sharp convergence zones coincides with the plume boundary nearly perfectly. The thick lines start to separate from the plume after three hours of evolution (initially at the northern periphery), and this process of wave detachment is mostly completed during the next two hours.

[21] To make sure that the thick lines moving off the plume really represent IWs, let us consider the vertical structure of the salinity field. Figure 5 illustrates several salinity cross sections at different stages of the plume evolution along the line $A-A$ shown in Figure 4. Scrutiny of Figure 5 reveals that the plume itself together with the mixed underlying waters occupies 10-meter surface layer, and the head of the gravity current is well developed by time span $t=2 \mathrm{~h}$ (see Figure 5, top). The nonlinear waves start to separate from the main body of the plume two hours later, and by $t=5 \mathrm{~h}$ they have already detached from the plume. Starting from this moment of time a packet of shortscale internal waves is clearly seen. Presumably, this is a consequence of the transition from supercritical to subcritical regime of the plume evolution taking place between 4 and 5 hours when arrested IWs are released and radiate from the plume.

[22] The density sections shown in Figure 6 illustrate three stages of wave evolution described by Nash and Moum [2005]. They are: (I) growth of the head of the gravity current, (II) wave fission resulted in the formation of internal waves, and finally, (III) the detachment of the wave packet from the plume and its free propagation. All these stages are clearly seen in Figure 6: at $4 \mathrm{~h}: 00 \mathrm{~min}$ (I) the head of the gravity current is developed; at $4 \mathrm{~h}: 20 \mathrm{~min}$ (II) the nonlinear wave separates from the main body of the plume and starts to evolve into a series of rank-ordered IWs. The wave packet propagates faster than the plume (quantitative estimates are presented below), and by $4 \mathrm{~h}: 40 \mathrm{~min}$ (III) it has moved several hundreds meters off.

[23] Figures 6(IV) and 6(VI) confirm that the fragments detached from the plume can be treated as internal waves: the structure of the isopycnals along with the horizontal and vertical velocities represent the first baroclinic mode. It is seen that the first wave in the packet is the largest wave with the amplitude of about $10 \mathrm{~m}$ and the wavelength of approximately $200 \mathrm{~m}$. The maximum horizontal and vertical velocities in this wave are $0.5 \mathrm{~m} \mathrm{~s}^{-1}$ and $0.04 \mathrm{~m} \mathrm{~s}^{-1}$, respectively (see Figure 6 for the location). The subsequent waves are weaker, and the packet itself is well rank ordered.

[24] The analysis of the BCR has shown that the waves start to separate from the southern and northern peripheries of the plume earlier than from its central sector. All the details on the wave detachment are presented in Figure 7 
salinity (ppt)

$\begin{array}{lllllllllllll}20 & 19 & 18 & 17 & 16 & 15 & 14 & 13 & 12 & 11 & 10 & 9 & 8\end{array}$
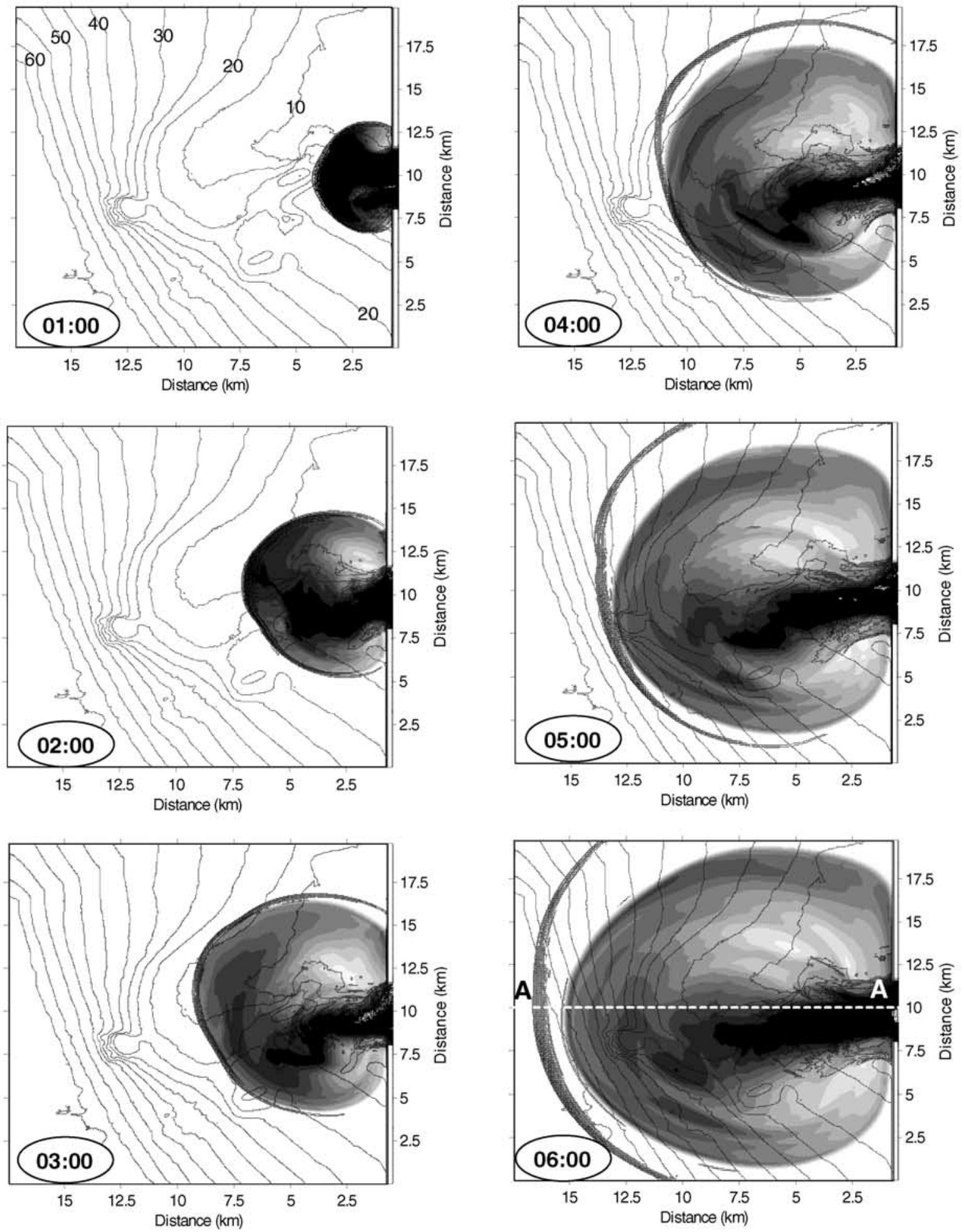

Figure 4. Time series presenting the surface salinity (gray for fresh water and white for saline water) overlaid with the maximum gradient of horizontal velocity $\left(G>10^{-3} \mathrm{~s}^{-1}\right.$, black lines) and bottom topography. 
salinity (ppt)

$\begin{array}{lllllllllll}33 & 30.5 & 28 & 25.5 & 23 & 20.5 & 18 & 15.5 & 13 & 10.5 & 8\end{array}$
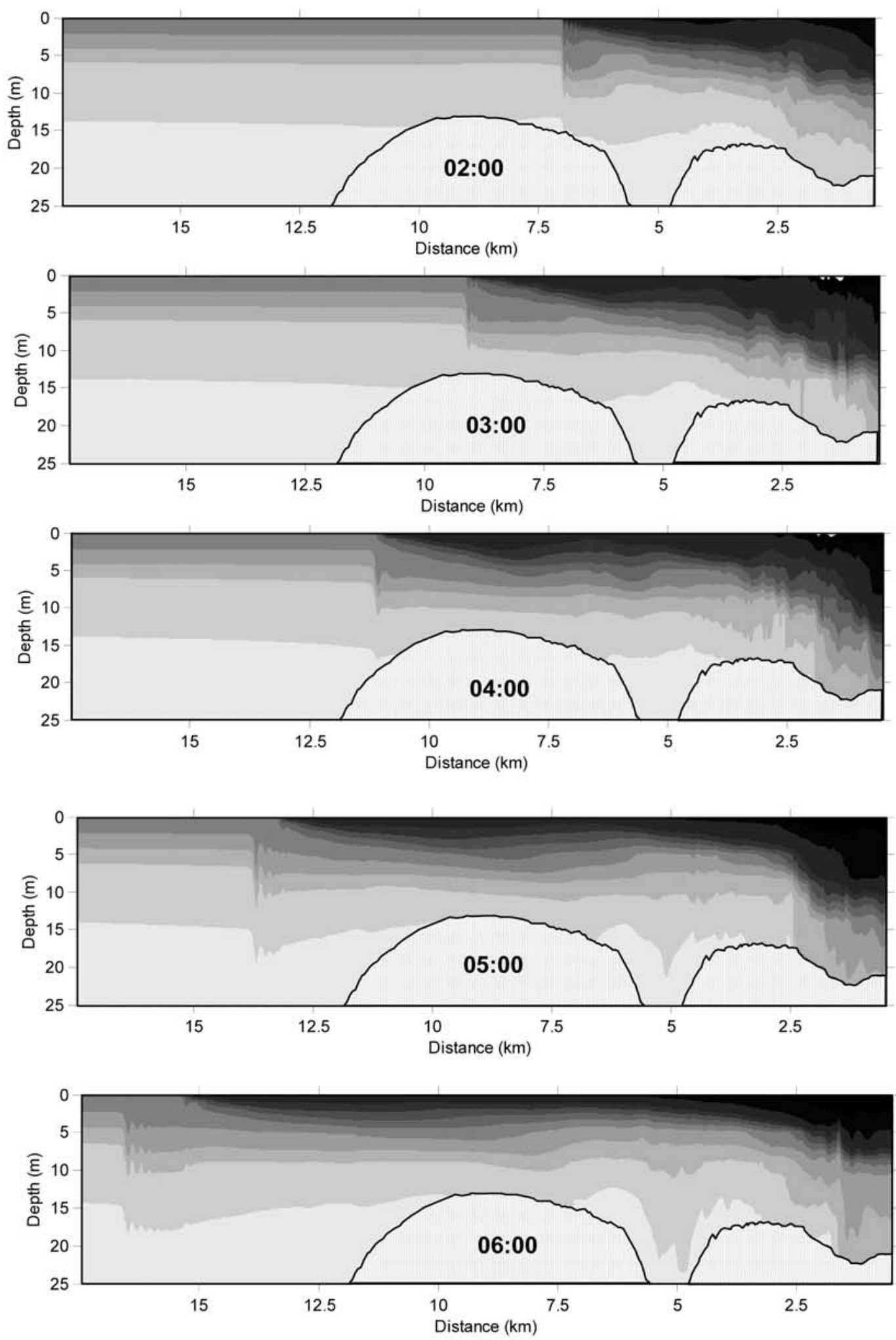

Figure 5. Evolution of the salinity field in the cross section A-A (see Figure 4). 

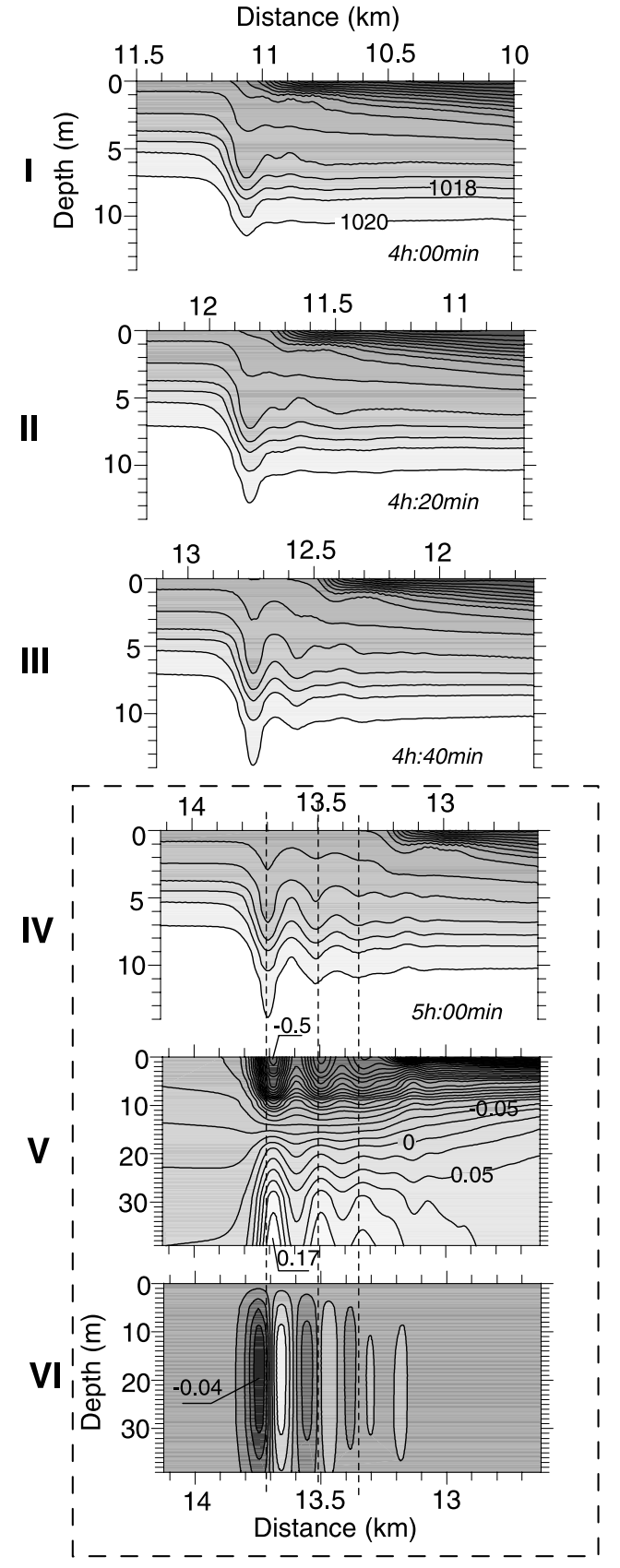

Figure 6. Density sections showing three stages of wave generation: (I) frontal growth, (II) wave fission, and (III) free wave propagation. Dashed rectangle: (IV) additional density section with corresponded (V) horizontal and (VI) vertical velocities (contour labels in $\mathrm{m} \mathrm{s}^{-1}$ ).

where the position of the plume boundary is shown with 10-minute interval. From Figure 7, one can estimate the velocity of the plume spreading, $U_{p}$. The dotted line represents the contour where the Froude number, $F r=U_{p} / c$, is equal to one, that is, $F r=1$ (here $c$ is the phase speed of linear long internal waves). The phase speed $c$ was calculated using the boundary value problem.

$$
q_{z z}+(N(z) / c)^{2} q=0, \quad q(0)=q(-H(x, y))=0,
$$

where $q(z)$ is the vertical wave profile, $N(z)$ is the buoyancy frequency and $H(x, y)$ is the water depth. To the left of the dotted line $F r<1$ whereas $F r>1$ to the right of it. According to the linear theory this line formally separates two areas with "subcritical" and "supercritical" regimes of the plume evolution. The dashed line shows the location of the wave detachment defined from the numerical experiment. In fact, this line specifies the actual boundary between subcritical and supercritical regions of the plume evolution.

[25] Several conclusions can be drawn from Figure 7. The first one is similar to those found for Figure 4, that the wave detaches earlier from the plume in the northern and southern peripheries than it happens in its central sector. The reason is that the plume propagates more slowly at the lateral boundaries than along the axis of the jet (compare the distance between contours in various parts of the calculation domain). To be more specific, the waves start to separate from the plume periphery when the speed of the plume spreading drops below $0.5 \mathrm{~m} \mathrm{~s}^{-1}$. This velocity found from Figure 7 coincides with the value found by Nash and Moum [2005]. At the same time in its central sector (in the direction where the majority of river water is accumulated) the plume propagates faster and the subcritical conditions for the waves are met here substantially later (up to 5 hours after start of experiment). As a result, the arrested internal

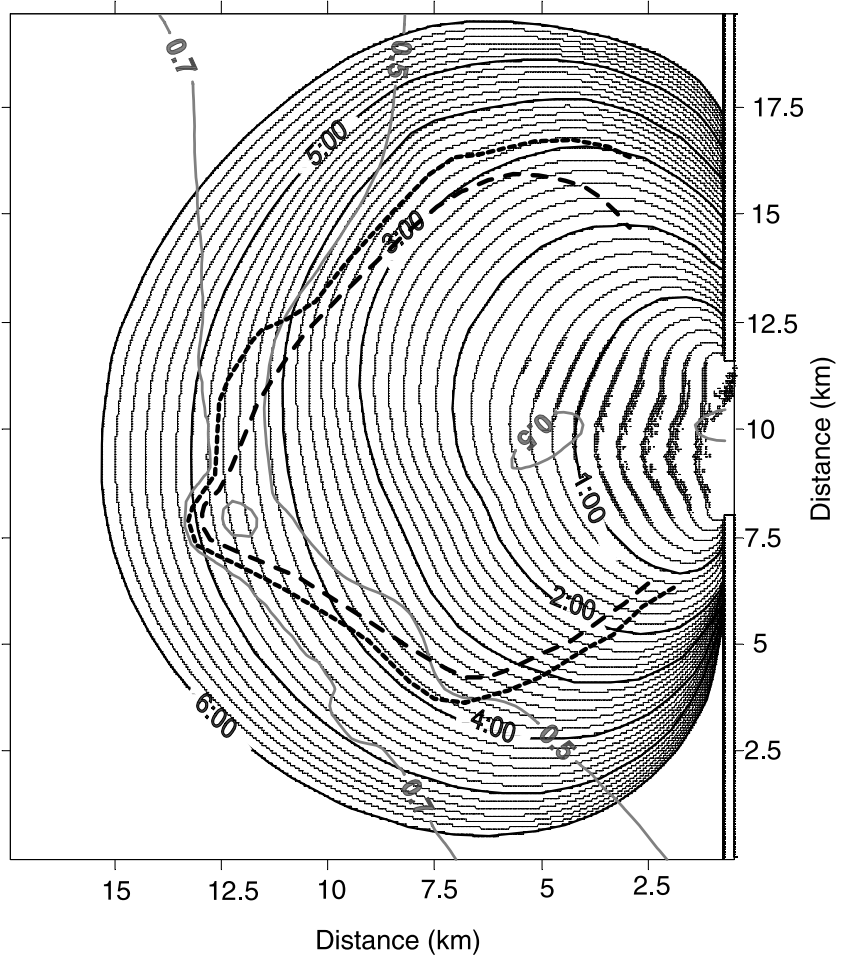

Figure 7. The position of the plume boundary presented with a 10-min interval (black thin and thick lines). Two thick gray lines show the contours of the linear phase speed in $\mathrm{m} \mathrm{s}^{-1}$. The thick dashed line shows the locations of the actual wave separation from the plume. The dotted curve indicates the positions of $F r=1$ calculated from linear boundary value problem (1). 

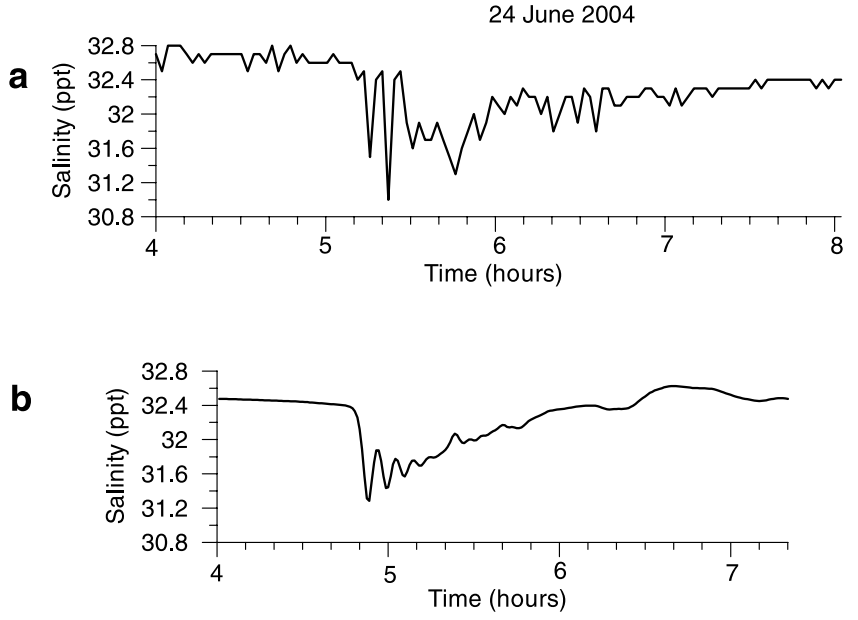

Figure 8. (a) Salinity measured by station OGI02 at 10-m depth. (b) Model predicted salinity at the same location.

waves accumulate more energy and thus their amplitude are greater here than amplitude of IWs generated at the periphery.

[26] Another important conclusion from Figure 7 is that the wave separates from the plume earlier than it was predicted by the linear theory (compare the position of the dashed and dotted curves). The difference can be explained in terms of nonlinear dispersion: large amplitude internal waves propagate faster than linear waves so that the gap between the two lines is just an illustration of this fact.

[27] Figure 8 represents the salinity recorded by mooring OGI02 at the depth of $10 \mathrm{~m}$ (Figure $8 \mathrm{a}$ ) and the salinity obtained for the same location numerically (Figure $8 \mathrm{~b}$ ). Both graphs reveal the characteristics of a "classical" solibore, that is, initial stage of disintegration of a baroclinic bore (the head of the plume, in fact), into a packet of solitary internal waves. The newborn rank-ordered packet of internal waves (the high-frequency oscillations) is clearly seen on the background of a sharp steplike structure of a smoothed salinity profile. The experimental profile is not as regular and rank ordered as the numerical graph. This fact can be explained by a relatively large sampling interval at OGI02, 2 min, which gives only three readings for a spike so that the absolute maximum of every can be missed. Another reason could be the interference of IWs with the background internal waves of other nature which (see Figure 1) are not included into the model. Taking this reasoning into account one can conclude that the both profiles qualitatively are in a good agreement. Even their quantitative details are basically considered: the vertical scope is in the range $\Delta S=1.6-1.2 \mathrm{ppt}$ and the distance between the picks is of about $10 \mathrm{~min}$ ).

\section{Sensitivity Runs}

[28] The BCR discussed above was performed for the three major driving forces acting together: (1) the density gradient between the estuary and the ocean, (2) the river discharge, and (3) the ebb tidal flow. Further investigations are needed to clarify the specific input of every force in the generation mechanism of IWs. For this purpose three extra "ad hoc" situations are considered below in addition to BCR when one or two driving forces are excluded from the analysis. Figure 9 presents four different scenarios of the plume evolution: the plume is initiated by the density gradient alone (Figure 9a), together with the river discharge (Figure 9b) or with tide (Figure 9c). Panel (d) shows the BCR for the comparison. The initial density contrast in this series of runs was identical: $\rho_{e}=1001.92 \mathrm{~kg} \mathrm{~m}^{-3}$ in the estuary; in the sea the density varies from $\rho_{s}(0)=1013 \mathrm{~kg}$ $\mathrm{m}^{-3}$ at the surface to $\rho_{s}(H)=1026 \mathrm{~kg} \mathrm{~m}^{-3}$ at the bottom. The river discharge was taken equal to $Q=5000 \mathrm{~m}^{3} \mathrm{~s}^{-1}$ and the tidal flow in the mouth was $U_{\text {tide }}=0.5 \mathrm{~m} \mathrm{~s}^{-1}$.

[29] Figure 9 shows that the propagating plume generates IWs in all scenarios. The plume size and shape, as well as the parameters of the radiated IWs are however highly sensitive to the applied force. The overall scale of the plume lens is larger and the generated IWs are stronger in the case of two or three driving forces rather than one only. Note also that without tide (Figures 9a and 9b) the lens of fresh water occupies twice smaller area than that formed with the tide (Figures 9c and 9d). It is evident that, being accompanied by the tidal current, the plume propagates faster. As a result, the transition from supercritical to subcritical regime of the plume evolution starts earlier when it is not accelerated by the tide and therefore the generated IWs moved off the plume much farther in scenarios (a) and (b) than in scenarios (c) and (d).

[30] Scrutiny of Figure 9 has shown that the plume initiated by the density gradient alone (without tide and river discharge) is however able to produce remarkable IWs (Figure 9a). The next series of experiments was aimed to test the sensitivity of the model to the value of the initial density gradient between the estuary and the sea. Note that there are two parameters that control the density gradient. The first one is the total density difference between the estuary and the sea, $\Delta \rho(z)=\left(\rho_{e}-\rho_{s}(z)\right)$. Taking into account the fixed water stratification at the shelf (the vertical profiles presented in Figure 1c), this parameter can vary only by changing $\rho_{e}$. In all experiments discussed above the density in estuary $\rho_{e}$ was equal to $1001.92 \mathrm{~kg}$ $\mathrm{m}^{-3}$ (almost fresh water), whereas $\rho_{s}(0)=1013 \mathrm{~kg} \mathrm{~m}^{-3}$. So, this density difference is acknowledged hereafter as a "large" difference. Another numerical experiment was performed for "small" density difference when $\rho_{e}=1010$ $\mathrm{kg} \mathrm{m}^{-3}$ (which was increased by taking $20 \mathrm{ppt}$ for salinity in the estuary).

[31] The second parameter which can change the value of the density gradient is the thickness of the transition zone, $\Delta L$, between two water bodies. It is clear that the sharp interface with $\Delta L=25 \mathrm{~m}$ produces a hydraulic jump at the leading edge of the gravity current almost immediately after the release of the lock, whereas the smoothed transition zone requires time to arrange similar frontal conditions. In addition to the "sharp" case with $\Delta L=25 \mathrm{~m}$, another numerical experiment was performed for the "smoothed" transition zone with $\Delta L=1 \mathrm{~km}$. Figure 10 represents three tested cases: sharp gradient $(L=25 \mathrm{~m})$ with large and small density difference are presented on Figures $10 \mathrm{a}$ and 10b, respectively; the case with small density difference between two water bodies and smoothed interface is presented by 


\section{$\begin{array}{lllllllllllll}20 & 19 & 18 & 17 & 16 & 15 & 14 & 13 & 12 & 11 & 10 & 9 & 8\end{array}$}
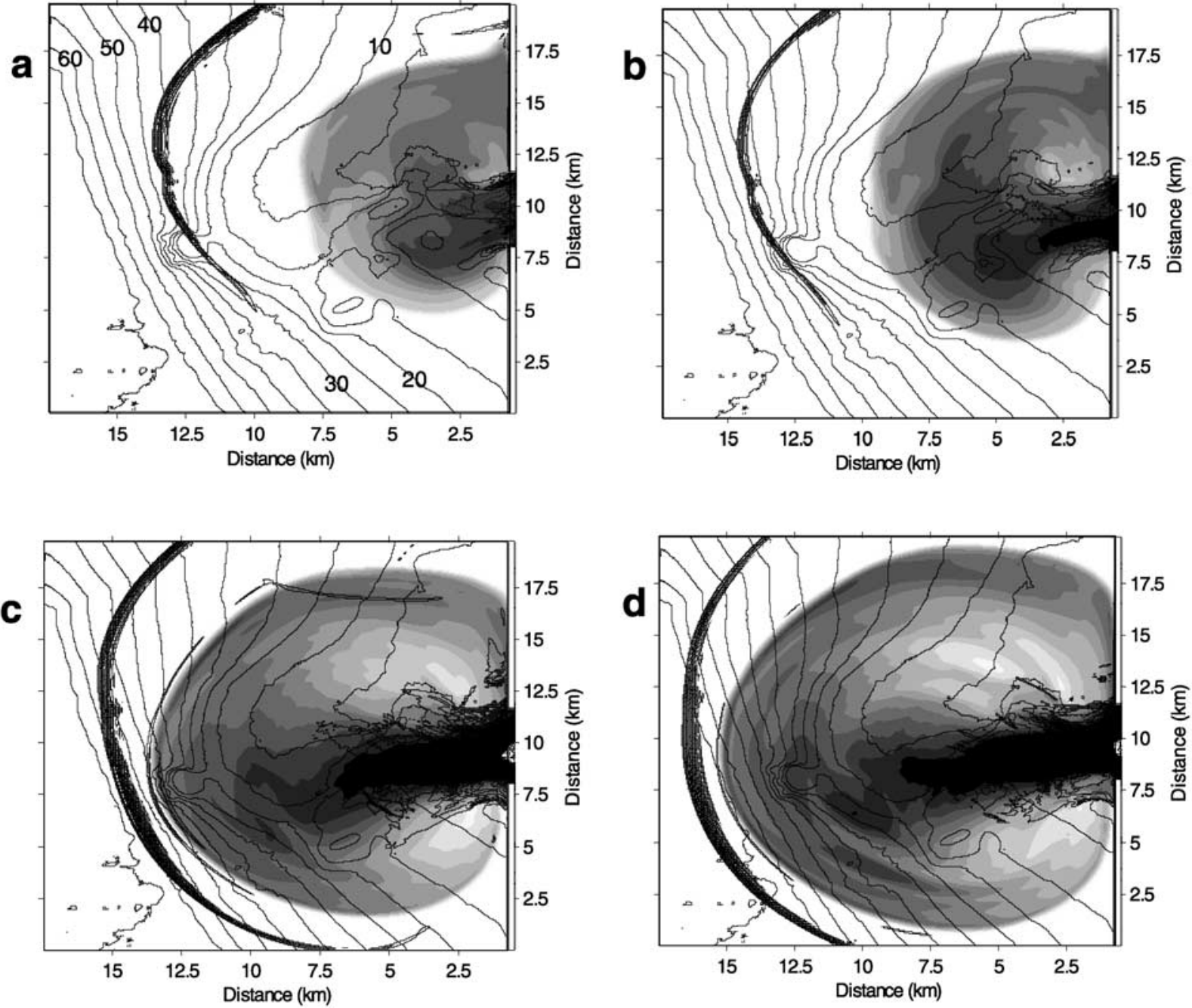

Figure 9. Surface salinity field after $t=6$ hours of the plume evolution overlaid with the gradient of horizontal velocity and bottom topography: (a) motion is initiated by the density difference between estuary and ocean; (b) the density gradient is accompanied by the river discharge; (c) the density gradient is accompanied by the tide; and (d) the density gradient, tide, and river discharge act together.

Figure 10c. The surface temperature is used here to trace the plume. It is overlaid with the bottom topography and horizontal velocity gradient $G \geq 10^{-3} \mathrm{~s}^{-1}$ (solid line) which indicate the position of IWs.

[32] It is seen from Figure 10 that the weaker density gradients, the more slowly propagates the plume. However, the density gradient alone (even smooth and weak) is still able to generate IWs. These waves are clearly seen in Figures 10b and 10c. Their amplitudes can be estimated from Figure 11 where the vertical density sections along the central axis of the plume (similar to Figures 4 and 6) are presented for the three stages of the wave generation. Panel A, B, C in Figures 10 and 11 correspond to each other.
[33] Inspection of Figure 11 reveals that the larger and sharper initial density gradient produces the strongest internal waves. Further decrease of the density gradient leads to a subsequent weakening of the wave signal, which can make difficult their experimental detection. However, an important outcome from these runs is that the river plume driving by the density gradient alone still generates internal waves, although much weaker than in the case when all three driving forces act together (compare Figures 6 and 11a).

[34] The results presented above have demonstrated the sensitivity of the plume evolution to the nature of the driving force: density gradient, river discharge or tidal 


\section{Temperature ${ }^{\circ} \mathrm{C}$}
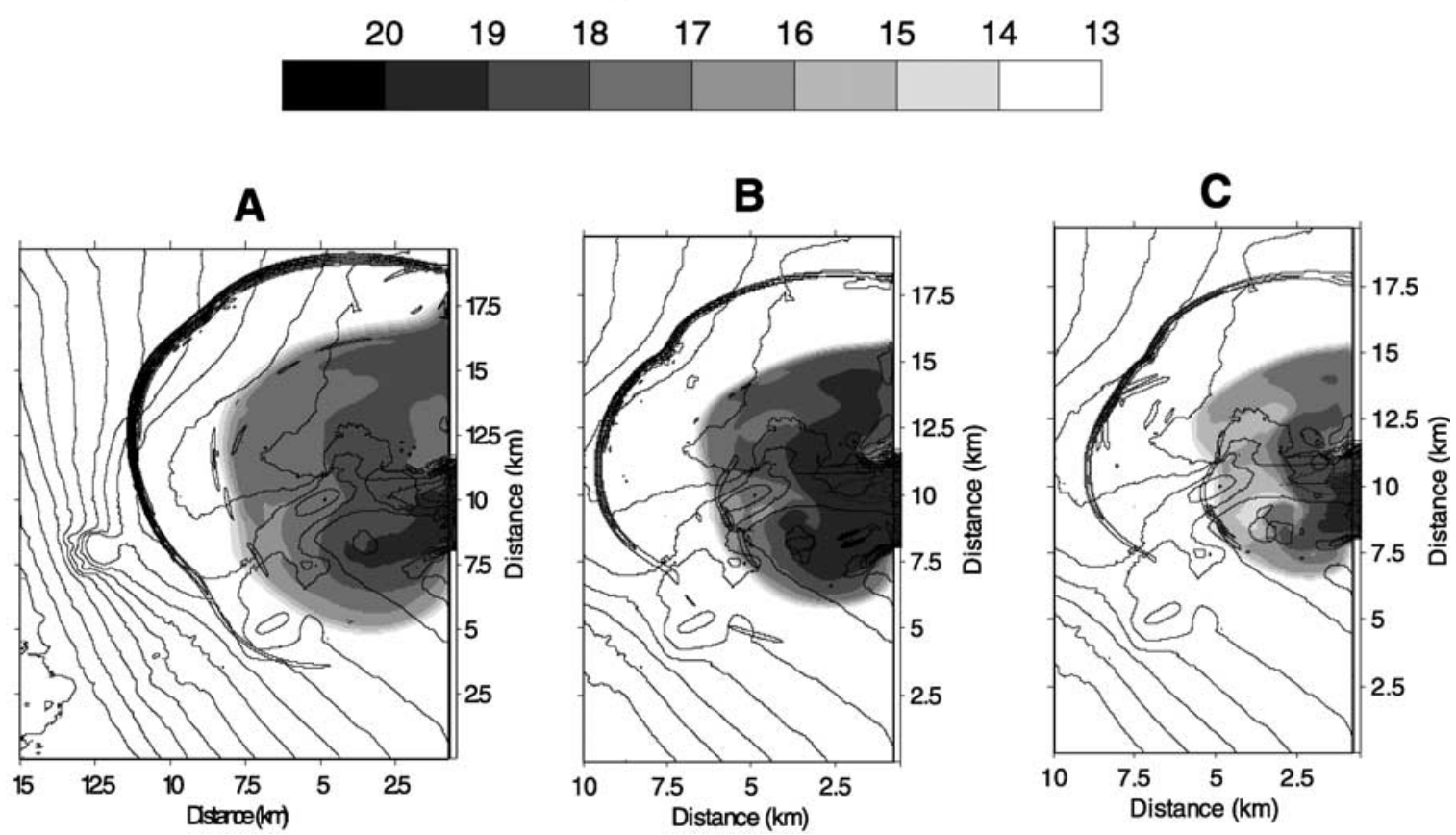

Figure 10. Surface temperature at $t=5$ hours overlaid with the gradient of horizontal velocity $G>$ $10^{-3} \mathrm{~s}^{-1}$ and bottom topography for different values of density gradient: (a) large and sharp, (b) small and sharp, and (c) small and smoothed (see explanation in text).

current. There are, however, two extra factors which are also important for the understanding of the plume dynamics. One of them is the effect of the errors introduced in the model by numerical discretization of the governing equa- tions. The coarser numerical grid can increase the numerical viscosity to such an extent that some important details of solution can be lost. In the present calculation, however, the model resolution was fine enough to prevent all undesirable

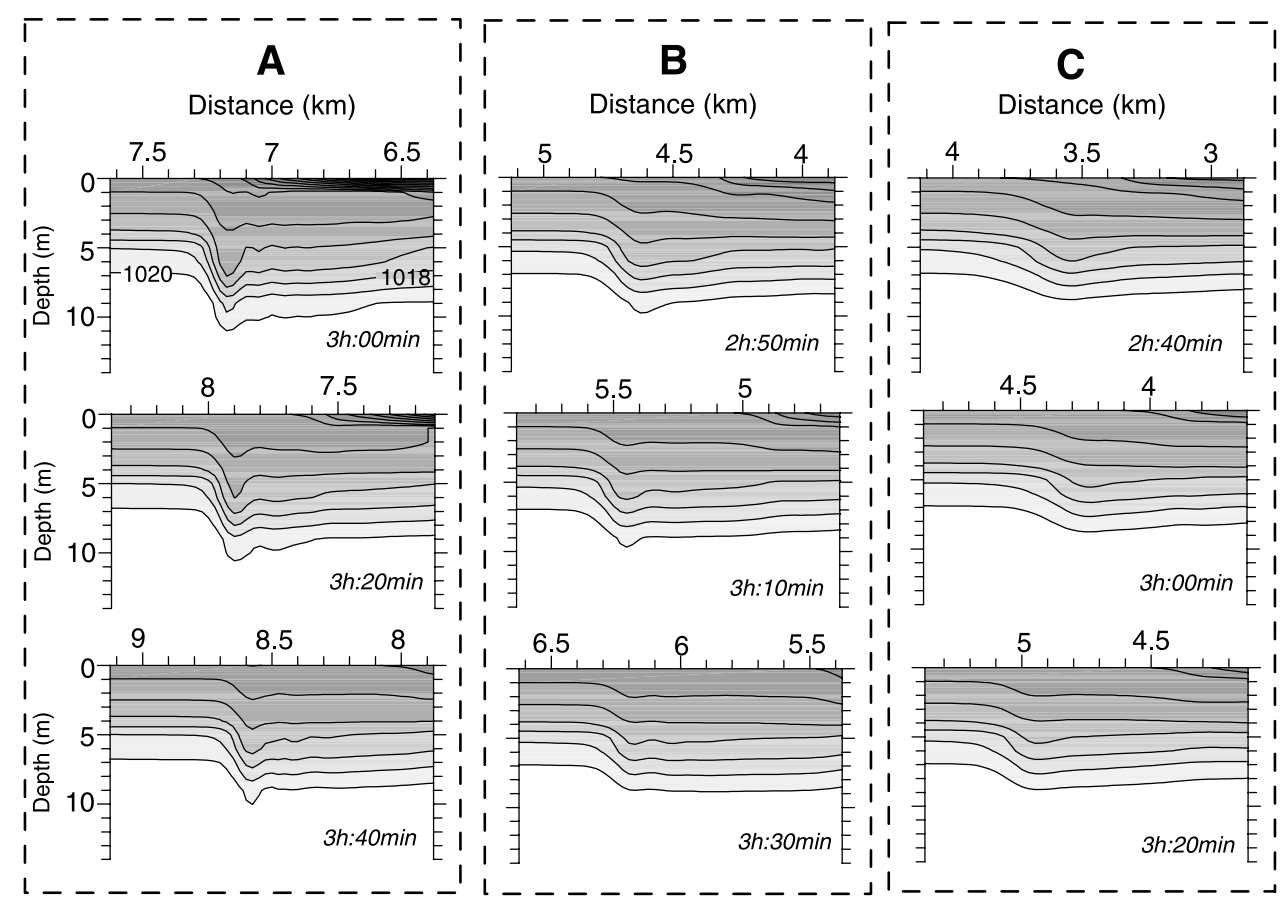

Figure 11. Density sections showing three stages of wave generation: frontal growth, wave fission, and free propagation for different values of density gradient: (a) large and sharp, (b) small and sharp, and (c) small and smoothed. 


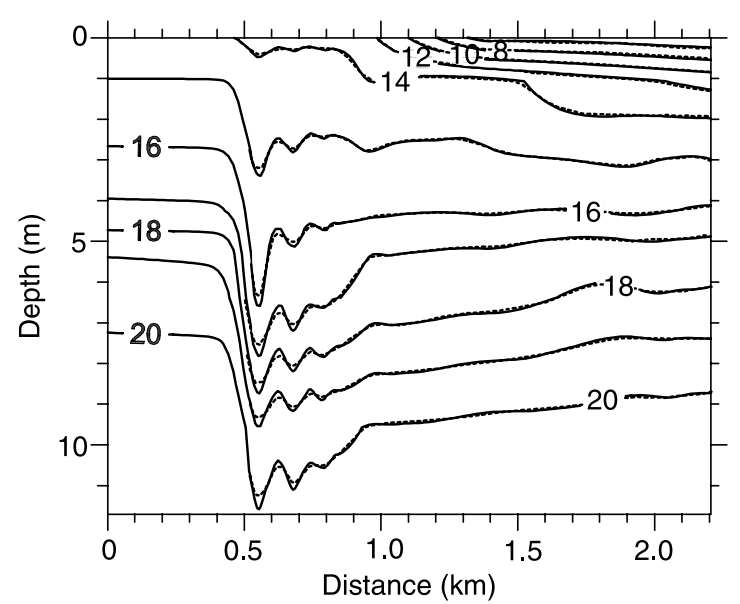

Figure 12. Density sections as in Figure 6 but calculated for the time span $t=4.5 \mathrm{~h}$ with $\Delta x=\Delta y=12.5 \mathrm{~m}$ (solid lines) and $\Delta x=\Delta y=25 \mathrm{~m}$ (dashed lines).

effects related to the numerical viscosity. Figure 12 clearly demonstrates that the amplitude of the leading waves is a bit larger on a finer grid. However the model with coarser grid still reproduces the oscillating solibore pretty successfully.

[35] Another factor which can affect the plume spreading is the shape of bottom topography. Even though the plume itself occupies only 10-meter-thick surface layer, it does "feel" the bottom topography although the total depth in the basic part of the model domain is greater than $20 \mathrm{~m}$ (see Figure 1b). As it was shown above the river waters turns to the right just after the leaving the mouth, which is in accordance with the Coriolis effect. However, the freshwater jet stream located in the middle of the plume, clearly turns to the left (see, for instance, Figure 4, time span $t=6 \mathrm{~h}$ ). It was assumed that the jet follows the general route of the underwater canyon. To investigate the role of the bottom topography its profile was simplified in such a way to be a flat slope from 10-m depth at the exit from estuary to $40 \mathrm{~m}$ at the western boundary of the calculation domain. The resulting salinity field for the time span $t=6 \mathrm{~h}$ of the plume evolution is presented in Figure 13 (overlaid with gradient velocity fields and bottom topography). The acting forces in this experiment were the same as in the BCR presented in Figure 4, that is, density gradient, tide, and river discharge. As it was expected, both the plume and the jet stream are curved to the right under the action of the Coriolis force. So, despite the fact that the freshwater plume spreads mostly in the thin surface layer, the plume itself is sensitive to the form of the bottom topography.

\section{Influence of Ambient Stratification}

[36] The idea of the generation of IWs by the river plume was formulated by Garvine [1984]. He considered a steady radial symmetric flow discharging from a point source of fresh water into a stationary ambient seawater. It was assumed that IWs are generated at the interface between two layers and propagate from the source toward the front. As soon as the waves reach the plume boundary they are reflected back into the interior of the plume where they are reflected back again from the area with reduced layer thickness and higher current speed. It is clear that the IWs could not overtake the front and radiate into the homogeneous fluid. It was further assumed that the waves arrested inside the plume can produce multiple fronts. To justify his idea Garvine [1984] presented several patterns of a radially symmetric river plume and acknowledged some other laboratory and field observations. Luketina and Imberger [1987] also discussed a possibility of generation of secondary fronts within the plume observed in the Koombana Bay. They did not support however the wave mechanism of their formation proposed by Garvine [1984] and attributed the subfronts generation to the variations of the river discharge. In fact, the water on the shelf beyond the Koombana Bay plume was well stratified, so a free radiation of the generated waves from the front was quite possible. For instance, in the context of the present results Figure 15 of [Luketina and Imberger, 1987] can be treated as an evidence of wave separation.

[37] It is demonstrated here that internal waves can be generated by a river plume, and this mechanism works in a broad range of the background conditions when three driving forces, that is, tidal flow, river discharge and horizontal density gradient act together, separately or in various combinations. Quite a typical situation for many estuarine systems is that the shelf water in the area of the contact of two water masses is well mixed (especially this concerns small-scale river plumes). As a result, no internal waves can be produced in homogeneous fluid, and the plume remains supercritical indefinitely. So, here we arrive at the conclusion that the water stratification on the shelf determines the conditions of internal wave radiation. It is demonstrated below that the most crucial parameter for
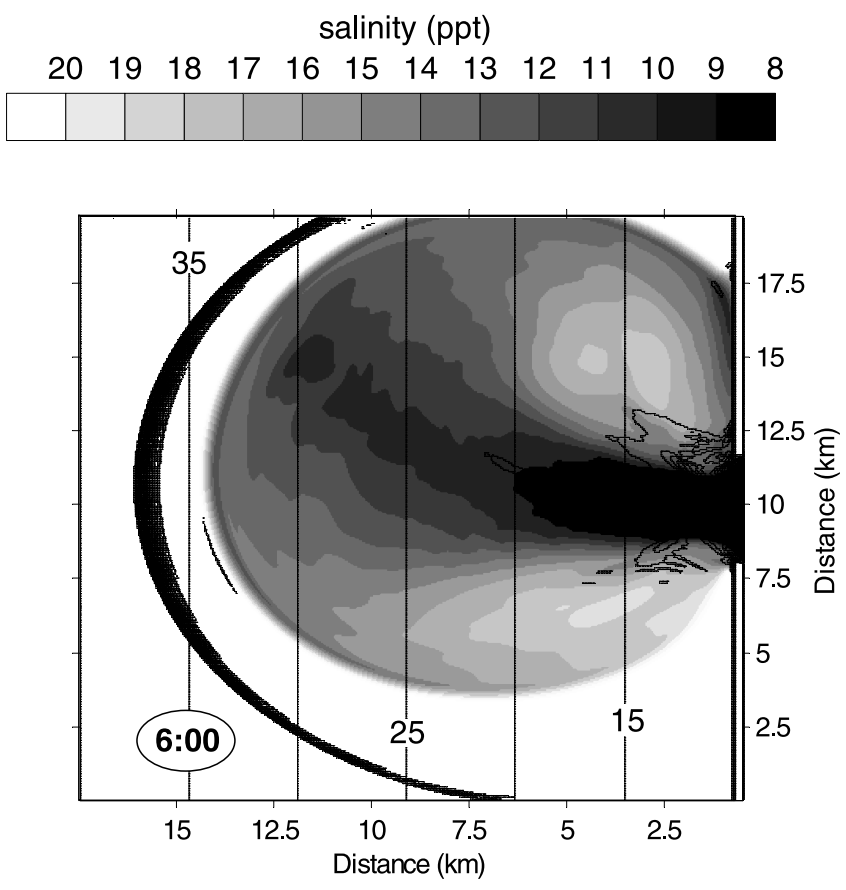

Figure 13. Surface salinity overlaid with the gradient of horizontal velocity and idealized bottom topography at $t=6 \mathrm{~h}$. 


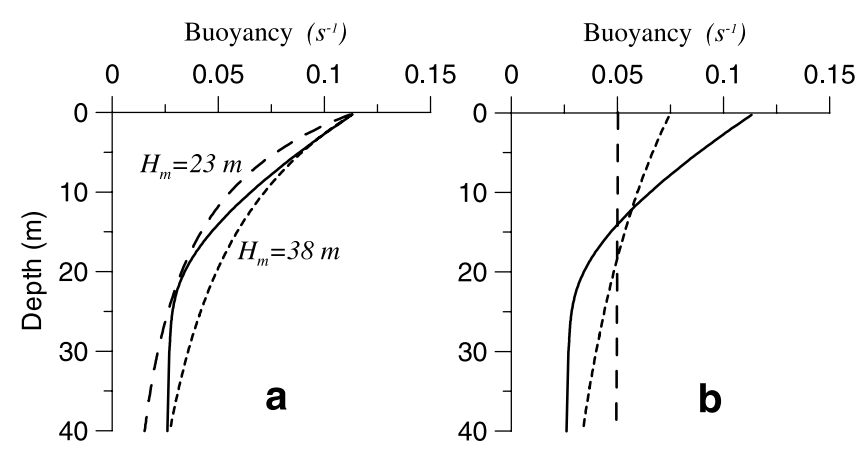

Figure 14. (a) The smoothed buoyancy frequency profile used in calculations (solid line) and two profiles defined by formula (2) for $H_{m}=23 \mathrm{~m}$ (dashed line) and $H_{m}=38 \mathrm{~m}$ (dotted line). (b) The buoyancy frequency profile used in Figure 16.

internal wave generation is the rate of changes of the buoyancy frequency with depth. This is a topic for the rest of this section.

[38] In some practical cases the following buoyancy frequency profile $N(z)$ is a pretty realistic approximation of the shelf water stratification:

$$
N(z)=\frac{N_{0}}{\left(1-z / H_{m}\right)^{2}} .
$$

This formula defines a decay of the buoyancy frequency with depth from its maximum value $N_{0}$ at the free surface $\left(H_{m}\right.$ is set positive and corresponds to the depth where $\left.N(z)=N_{0} / 4\right)$. For instance, the smoothed profile $N(z)$ in the area of the Columbia River plume is presented in Figure $14 \mathrm{a}$ by a solid line. The dashed and doted lines in the same graph represent profile (2) for $H_{m}=23 \mathrm{~m}$ and $38 \mathrm{~m}$, respectively. Figure $14 \mathrm{a}$ demonstrates that with some accuracy formula (2) interpolates experimental curve quite satisfactory.

[39] An analytical solution for internal solitary waves (ISW) radiated from the plume and propagating in the fluid with stratification (2) can be found similarly to the procedure described by Vlasenko et al. [2005, section 5.2]. Without the loss of generality a two-dimensional case is considered here so that the stream function $\Psi(x, z, t)(u=$ $\partial \Psi / \partial z$ and $w=-\partial \Psi / \partial x)$ can be introduced. The Long's equation for IWs of permanent form propagating with velocity, $V$, with appropriate boundary conditions takes the form:

$$
\Psi_{\theta \theta}+\Psi_{z z}+\frac{\Psi}{V^{2}} N^{2}\left(z-\frac{\Psi}{V}\right)=0, \quad \Psi_{\theta}=0, z=0 ; z=-H
$$

where $\theta=x-V t$. With new variables $\zeta=\theta / \lambda, \eta=z / H, F=$ $\Psi / V H$ system (3) reads

$$
\varepsilon^{2} F_{\zeta \zeta}+F_{\eta \eta}+\beta F \hat{N}^{2}(\eta-F)=0, \quad F_{\zeta}=0, \eta=0, \eta=-1 .
$$

Here $\varepsilon=H / \lambda, \hat{N}(\eta)=N(z) / N_{0}$ and $\beta=\left(H N_{0} / V\right)^{2}\left(\lambda\right.$ and $N_{0}$ are the wavelength and the scale of the buoyancy frequency, respectively). Assuming weak dispersion, $\varepsilon \ll$ 1 , the solution can be presented in terms of asymptotic expansions as follows:

$$
\begin{aligned}
F(\zeta, \eta) & =\varepsilon^{2} F_{1}+\varepsilon^{4} F_{2}+o\left(\varepsilon^{4}\right) \\
\beta & =\beta_{0}+\varepsilon^{2} \beta_{1}+\varepsilon^{4} \beta_{2}+o\left(\varepsilon^{4}\right) .
\end{aligned}
$$

Assuming that $F_{1}(\zeta, \eta)=\Phi(\eta) M(\zeta)$, in the first-order problem (4) splits into two: integrated Korteweg-de Vries equation for spatial function $M(\zeta)$

$$
\begin{aligned}
& M_{\zeta \zeta}+\gamma M^{2}+\beta_{1} \alpha M=0 ; \quad \alpha=\frac{\int_{-1}^{0} \bar{N}^{2} \Phi^{2} d \eta}{\int_{-1}^{0} \Phi^{2} d \eta} \\
& \gamma=-\beta_{0} \frac{\int_{-1}^{0} \Phi^{3} \frac{d \bar{N}^{2}}{d \eta} d \eta}{\int_{-1}^{0} \Phi^{2} d \eta}
\end{aligned}
$$

and boundary value problem for the definition of the vertical structure function, $\Phi(\eta)$

$$
\Phi_{\eta \eta}+\beta \Phi \hat{N}^{2}(\eta)=0 ; \quad \Phi(0)=\Phi(-1)=0,
$$

In summary, the solution of problem (3) in the first-order presenting the ISW of depression with wave amplitude $A$ for the stratification (2) reads

$$
\Psi(x, z, t)=-\frac{A V}{\Phi_{\max }} \Phi(z) \cosh ^{-2}\left(\sqrt{-\frac{A \gamma}{6 H^{3} \Phi_{\max }}}(x-V t)\right) .
$$

Here $\Phi(z)=(b \eta+1) \sin (\pi \varphi), b=-H / H_{m}, \Phi_{\max }=$ $\max \mid(\Phi(z) \mid$ and $\varphi=\eta(b-1) /(b \eta+1)$. All other parameters are as follows: $V=N_{0} H / \sqrt{\beta+2 A \gamma /\left(3 \alpha H \Phi_{\max }\right)}$ is the nonlinear phase speed of ISW, and $\beta=\pi^{2}(b-1)^{2}$.

[40] Taking into account that the surface gradient of the horizontal velocity is used for the detection of IWs, the next step is to differentiate formula (5) with respect to $z$ and $x$ in order to find the maximum of the horizontal velocity gradient, $\max |\partial u / \partial x|$ at $z=0$. In terms of the parameters introduced above this function reads

$$
\max \left|\frac{\partial u}{\partial x}\right|_{z=0}=\frac{4}{9} \pi N_{0}^{2} \sqrt{\frac{2 A^{3}}{H^{3}}} f(b)
$$

where $f(b)$ depends on only one parameter $b=-H / H_{m}$ :

$$
f(b)=\sqrt{\frac{b(b-1)^{2}}{\Phi_{\max }^{3}} \frac{\int_{-1}^{0}(b \eta+1)^{-2} \sin ^{3}(\pi \varphi) d \eta}{\int_{-1}^{0}(b \eta+1)^{2} \sin (\pi \varphi) d \eta}}
$$

Note that for the simplicity the nonlinear correction to the phase speed $V$ was neglected.

[41] It is important that the function $f(b)$ does not depend on the plume characteristics. In the broad range of the parameter $b$ (from $-\infty$ to 0 ) it describes a transition from a sharp decrease of $N(z)$ with depth when $b \rightarrow-\infty\left(H_{m} \rightarrow\right.$ 0 ) to almost monotonous stratification, $N(z) \rightarrow$ const when 


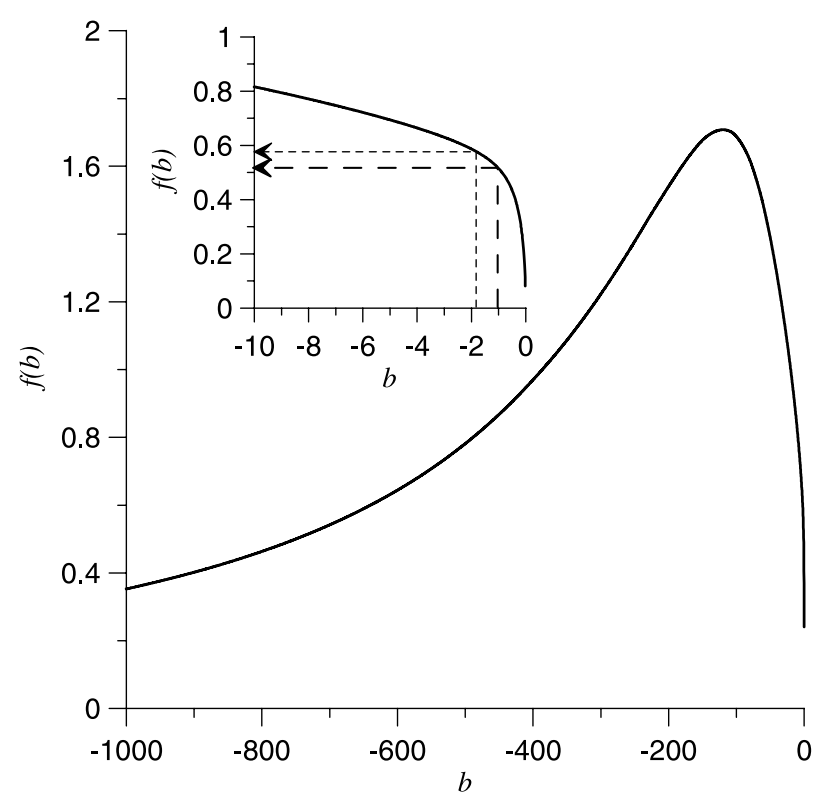

Figure 15. The graph of function $f(b)$.

$b \rightarrow 0$. Formula (6) can be used for quick estimation of the intensity of backscattered signal to realize whether the waves radiated from plume can be recorded by SAR. The example below illustrates the procedure.

[42] Figure 14a presents two $N(z)$ profiles (2) (dashed and dotted lines) along with the smoothed experimental curve (solid line) used in the model. Obviously, formula (2) cannot give the best fit for the experimental curve. Hence it is reasonable to consider the range of parameter $b$ which covers the variation of the experimental profile. The coefficient $b=-H / H_{m}$ for the dashed and doted lines in Figure 14a is equal to -1.74 and -1.05 , respectively. Appropriate values of the function $f(b)$ are 0.58 and 0.53 , accordingly (dotted and dashed line in Figure 15). It is clear that function $f(b)$ is not sensitive to the error in definition of $b$ when $b<$ -1 . However, the estimations of $b$ must be extremely accurate when the stratification is close to the monotonous (when $b>-0.5$, see Figure 15).

[43] The final step of the procedure is the substitution of the maximum value of the buoyancy frequency $N_{0}=$ $0.114 s^{-1}$ and the calculated values of $f(b)$ into (6). The only parameter which remains unknown yet is the amplitude of IWs. As it was shown above, the maximum amplitude of the leading internal wave is comparable with the vertical scale of the head of the gravity current. This value can be used as a measure for IW amplitude. Final calculation for $A=$ $10 \mathrm{~m}$ shows that the maximum value of the gradient of horizontal velocity $u$ at the free surface, $\max \left|\frac{\partial u}{\partial x}\right|_{z=0}$, lies in the range $(1.70 \div 1.86) \cdot 10^{-3} \mathrm{~s}^{-1}$ which is well above the threshold of $10^{-3} \mathrm{~s}^{-1}$ to be recoded by SAR [Alpers, 1985].

[44] Let us analyze an extreme case of monotonous fluid stratification. If $H_{m} \rightarrow \infty$ formula (2) transforms into $N(z)=$ $N_{0}=$ const, and consequently $b=0, \beta_{0}=\pi^{2}, \gamma=0$. The expression for the phase speed $V$ is simplified to $V=N_{0} H / \pi$. It is clear also that the coefficient of quadratic nonlinearity $\gamma=0$ for $N(z)=$ const so that the wavelength $\lambda$ in (5) is equal to infinity. This means that we cannot expect generation of internal solitary waves defined by (5) if plume intrudes into monotonously stratified water. The only signal which can be expected is a series of linear internal waves. Probably this type of waves was observed in the experiments by Maxworthy at al. [2002], who investigated the dynamics of a decelerating bottom gravity current produced in a lock release experiment. In this experiment the radiation of the upstream propagating internal waves were really observed in linearly stratified fluid although only for a very short time span.

[45] To understand the influence of the water stratification on the generation of IWs by the plume, two additional series of numerical experiment were performed. The both new buoyancy frequency profiles are shown in Figure 14b. One of them with $N(z)=$ const $=0.051 \mathrm{~s}^{-1}$ represents the monotonously stratified fluid with the linear increase of density with depth (dashed line in Figure 14b). The other numerical experiment was performed for buoyancy frequency defined by (2) with parameters $N_{0}=0.074 \mathrm{~s}^{-1}$ and $H_{m}=$ $81 \mathrm{~m}$ (dotted line in Figure 14b), which can be considered as a transitional case between real and monotonous stratifications. The original smoothed experimental profile $N(z)$ is also shown in Figure $14 \mathrm{~b}$ (solid line). Note that the density difference between the free surface and the bottom coincide in all cases. The driving forces in this experiment, that is, the density gradient and the river discharge are similar to that presented in Figure 9b: $\rho_{e}=1001.92 \mathrm{~kg} \mathrm{~m}^{-3}$, $\rho_{s}(0)=1013 \mathrm{~kg} \mathrm{~m}^{-3}, \rho_{s}(H)=1026 \mathrm{~kg} \mathrm{~m}^{-3}$ and $Q=$ $5000 \mathrm{~m}^{3} \mathrm{~s}^{-13}$. The results of the three runs are shown in Figure 16: the Figure 16 (left) represents the plume structure developing in real oceanic water (solid line in Figure 14b), the Figure 16 (middle) corresponds to the dotted profile in Figure 14b, whereas the Figure 16 (right) shows similar pictures for $N(z)=$ const (dashed line in Figure 14b). The plan view of the plume is given here along with series of vertical cross sections.

[46] Comparison of the surface signatures of IWs of Figures 16a, 16e, and 16i; 16b, 16f, and 16j; and 16c, $16 \mathrm{~g}$, and $16 \mathrm{k}$ shows that nonlinear wave structure separates from the plume $30 \mathrm{~min}$ later in the intermediate case and $1 \mathrm{~h}$ 20 min later in case of $N=$ const than it takes place for the real fluid stratification. Note also that initial internal bore at the moment of detachment has steep frontal face and gently sloping trial edge in all three cases (see Figures 16b, 16f, and 16j). However, its further evolution in the three considered cases is completely different. In the fluid with $N \neq$ const the leading nonlinear internal wave basically preserves its initial wavelength although the wave amplitude decreases slightly because of a radial divergence and energy transfer to the wave tail (see Figure 16d). Important is that the radiated internal wave disintegrates into a nonlinear wave packet which can be clearly identified during three hours after detachment (see Figures 16b, $16 \mathrm{c}$, and 16d) and later.

[47] The salinity sections in the intermediate case (Figures 16f, 16g, and 16h) show that even though the plume does radiate IW into the fluid with slowly decaying $N(z)$, this wave is wider than that for real $N(z)$ (because of smaller values of $\gamma$ ), and, in addition, it does not disintegrate into a wave packet.

[48] The detached baroclinic bore in the case of $N(z)=$ const (Figures 16j, 16k, and 161) gradually loses its compact shape and transforms into a small-amplitude long internal wave without nonlinear steepening and disintegration into a 


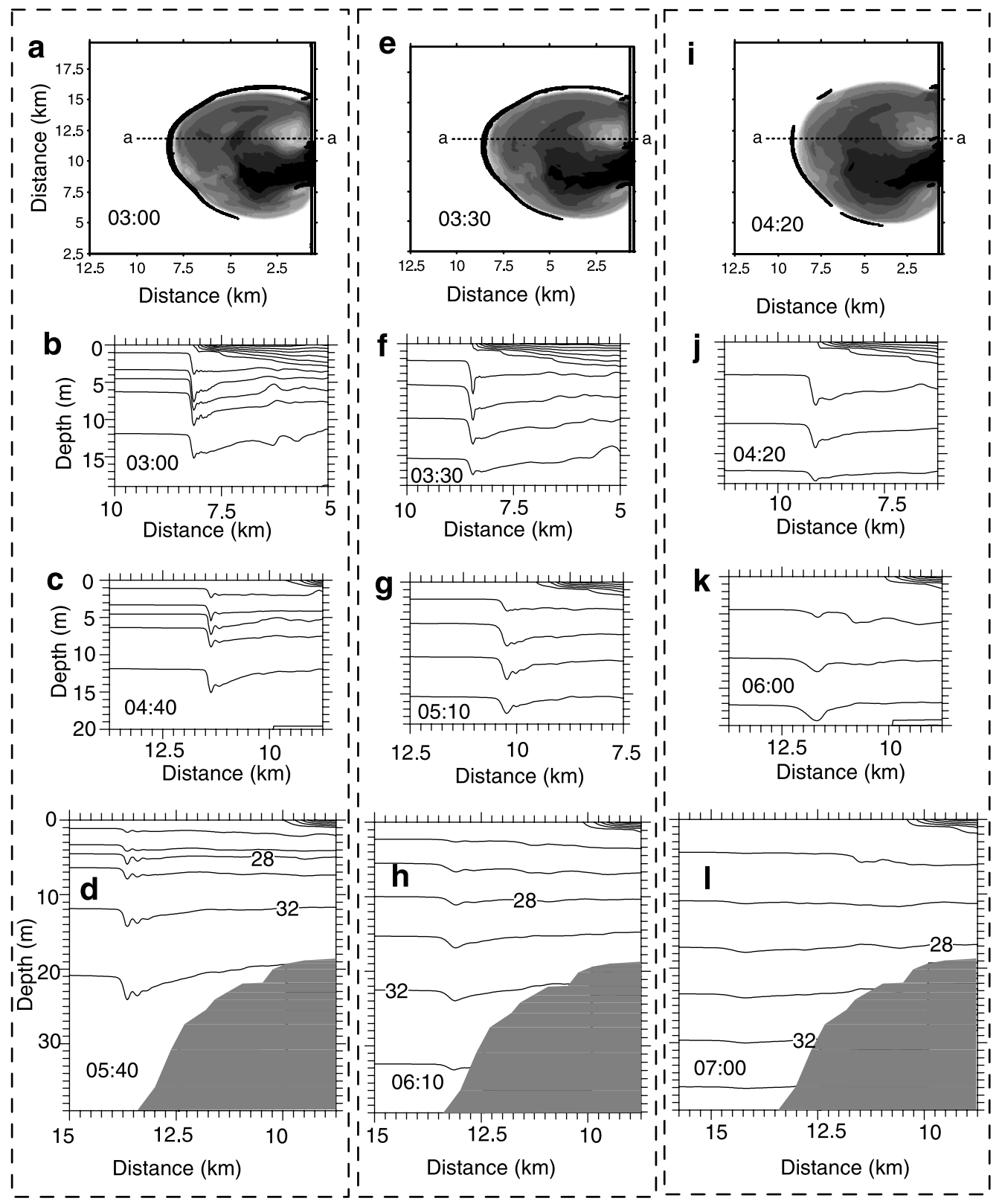

Figure 16. Internal wave generation for three types of stratification: (left) real $N(z)$ (solid line in Figure 14b), (right) linear stratification (dashed line in Figure 14b), and (middle) intermediate stratification (dotted lines in Figure 14b). Surface salinity overlaid with the gradient of horizontal velocity fields is shown at the top, and the three stages of wave evolution along salinity sections a-a (see upper panels a, e, i) are presented below.

packet of solitary internal waves. Such a behavior is a consequence of zero value of the quadratic nonlinearity coefficient $\gamma$ when $N(z)=$ const, hence without nonlinearity the initial disturbance in the monotonously stratified fluid evolves into small-amplitude long waves according to linear dispersion. Probably similar effect would be observed in the laboratory experiments by Maxworthy at al. [2002] if the tank was longer.

\section{Discussion and Summary}

[49] The observations performed by Nash and Moum [2005] revealed the evidence of IWs generated by the
Columbia River plume at the frontal edge of the gravity freshwater current that intrudes into the coastal saline water. Quite a reasonable explanation of their generation was a hypothesis that the wave separation from the decelerating front occurs when the plume speed falls below the local phase speed of propagation of IWs.

[50] The model investigations performed here allowed to study the process of river-sea interaction in a wide range of input parameters. In the BCR describing the typical oceanographic conditions with three driving forces acting together, that is, the ebbing tidal flow, river discharge and horizontal density gradient, it was found that the plume 
behaves as a jet. The fresh river water collides with the stagnant shelf waters and sinks down in the area of the plume leading edge forming a head of the gravity current. In supercritical conditions which are normally realized at the first stage of the ebb tidal phase the head of the gravity current contains a system of internal waves which are arrested inside the plume. In the course of propagation the plume decelerates because of the radial spreading.

[51] Another factor which contributes to the plume deceleration is the weakening of the ebb tidal current. Thus at a definite stage of the plume evolution its velocity $U_{p}$ falls below the wave phase speed, $V$. This is the condition for the separation of the head of the gravity current from the plume, that is, $U_{p}<V$, and its further disintegration into a packet of radiated internal waves. It should be noted that in the case of the Columbia River plume the radiated waves are strongly nonlinear. Taking into account that nonlinear waves propagate faster than linear ones, in definition of exact hydrodynamic conditions of internal wave separation the phase speed $V$ should not be calculated from the linear boundary problem (1), but has to include a nonlinear correction as well. For quick estimations the weakly nonlinear theory (similar to (3)-(5)) can be used as a starting point. As a result of the aforementioned, in reality internal waves can detach from the plume substantially earlier than it is predicted by the linear theory. Figure 7 illustrate this effect. This conclusion is in conformity with the results of the paper by Akylas [1984] and Grimshaw and Smyth [1986] where the resonant mechanism of internal wave generation at $F r \geq 1$ is discussed.

[52] Sensitivity runs performed for various combinations of driving forces acting in the area of the Columbia River plume result in the general conclusion: the buoyancy fluxes alone or in combination with other driving forces such as river discharge or tidal current are not able to provide necessary conditions for an efficient generation and radiation of internal waves. They are really important in a general energy balance because they control the intensity and number of internal waves emerged from the plume when this event really occurs. However, it was found that the background stratification of the ambient oceanic waters is the most important parameter for the wave generation by the plume. Not all buoyancy frequency profiles provide equal conditions. For instance, in monotonous stratified fluid $(N(z)=$ const $)$ the coefficient of quadratic nonlinearity $\gamma$ is equal to zero. Consequently, under the action of the dispersion alone the radiated wave transforms into a series of infinitesimal long waves (see Figure 16). In the limit of well mixed waters on the shelf, IW will never emerge from the plume. On the contrary, the dramatic decrease of the buoyancy frequency from the surface to the bottom provides necessary condition for the IWs generation. The importance of the seawater stratification for the generation process was also reported in observations by Pan and Jay [2008]. They found that the front-generated ISWs were recorded in about $25 \%$ of all available SAR images taken during upwelling events when shelf waters were well stratified. At the same time only a few SAR images taken during downwelling conditions reveal clear IW signal because shelf waters presumably were close to homogeneous.

[53] It is worth to note here that IWs are visible on images as systems of bright and dark parallel bands which are the results of modulation of surface waves caused by wind. According to the first-order radar imaging theory [Alpers, 1985] the relative deviation of the normalized radar cross section (NRCS) is linearly related to the gradient of the surface strain rate of internal waves $\partial u / \partial x$ as follows:

$$
\frac{\delta \sigma}{\sigma_{0}}=-(4+\mu) \tau_{r} \frac{\partial u}{\partial x}
$$

Here $\delta \sigma=\sigma-\sigma_{0}$ denotes the deviation of the normalized radar cross section, $\sigma$, from its mean value $\sigma_{0} ; \mu$ is the ratio between the group and phase velocity of the Bragg waves $(\mu=0.5$ for gravity waves, the relaxation time of surface waves $\tau_{r}$ should lie in the range 4.7-47 s). If $\partial u / \partial x \cong 10^{-3} \mathrm{~s}^{-1}$ and $\tau_{r}=40 \mathrm{~s}$ (for the wind of about $4 \mathrm{~m} \mathrm{~s}^{-1}$ ), then $\delta \sigma / \sigma_{0} \approx 0.2$, value commonly observed in radar imagery for internal waves.

[54] Formulas (2)-(6) along with Figure 15 can be used as a quantitative tool to answer the question when it can happen and whether these waves are sufficiently strong to be observed from space. The input parameters for these formulas are the maximum of the buoyancy frequency at the free surface, $N_{0}$, the depth of the fourfold buoyancy frequency decay, $H_{m}$, and the vertical scale of the head of the gravity current, $A$.

[55] Another finding from the present study is that the bottom topography can also play a remarkable role in the formation of the plume and especially in the generation of its surface "fine structure". In spite of the fact that the river plume is a pure surface phenomenon, that is, the freshwater lens in the shelf area occupies upper "skin" layer, the shape of the plume, its internal structure as well as the curvature of generated internal waves, time of their separation and the amplitudes depend not only on the stratification of the underlying waters and intensity of driving forces, but also on the bottom profile.

[56] Acknowledgments. This work was supported by NERC grant $\mathrm{NE} / \mathrm{E} 01030 \mathrm{X} / 1$. We thank two anonymous reviewers for their great attention to our paper.

\section{References}

Akylas, T. R. (1984), On the excitation of long nonlinear water waves by a moving pressure distribution, J. Fluid Mech., 141, 455-466.

Alpers, W. (1985), Theory of radar imaging of internal waves, Nature, 314, $245-247$.

Garrett, C., and E. Kunze (2007), Internal tide generation in the deep ocean, Annu. Rev. Fluid Mech., 39, 57-87.

Garvine, R. W. (1984), Radial spreading of buoyant, surface plumes in coastal waters, J. Geophys. Res., 89, 1989-1996.

Grimshaw, R., and N. Smyth (1986), Resonant flow of a stratified fluid over topography, J. Fluid Mech., 169, 429-464.

Fu, L. L., and B. Holt (1982), Seasat views oceans and sea ice with synthetic-aperture radar, JPL Publ. 81-120, Jet Propul. Lab., Pasadena, Calif.

Hickey, B. M., and N. S. Banas (2003), Oceanography of the Pacific Northwest coastal ocean and estuaries with application to coastal ecosystems, Estuaries, 26(48), 1010-1031.

Horner-Devine, A. R. (2008), The bulge circulation in the Columbia River plume, Cont. Shelf Res., doi:10.1016/j.csr.2007.12.012.

Luketina, D. A., and J. Imberger (1987), Characteristics of a surface buoyant jet, J. Geophys. Res., 92, 5435-5447.

Marshall, J., A. Adcroft, C. Hill, L. Perelman, and C. Heisey (1997), A finite-volume, incompressible Navier-Stokes model for studies of the ocean on parallel computers, J. Geophys. Res., 102, 5733-5752.

Maxworthy, T., J. Leilich, J. E. Simpson, and E. H. Meiburg (2002), The propagation of a gravity current into a linearly stratified fluid, J. Fluid Mech., 453, 371-394. 
Moum, J. N., D. M. Farmer, W. D. Smyth, L. Armi, and S. Vagle (2003), Structure and generation of turbulence at interfaces strained by internal solitary waves propagating shoreward over the continental shelf, J. Phys. Oceanogr., 33, 2093-2112.

Nash, J. D., and J. N. Moum (2005), River plumes as a source of largeamplitude internal waves in the coastal ocean, Nature, 437, 400-403.

Orton, P. M., and D. A. Jay (2005), Observations at the tidal plume front of a high-volume river outflow, Geophys. Res. Lett., 32, L11605, doi:10.1029/2005GL022372.

Pacanowski, R. C., and S. G. H. Philander (1981), Parametrisation of vertical mixing in numerical models of tropical oceans, J. Phys. Oceanogr., 11, 1443-1451.

Pan, J., and D. A. Jay (2008), Dynamic characteristics and horizontal transports of internal solitons generated at the Columbia River plume front, Cont. Shelf Res., doi:10.1016/j.csr.2008.01.002.

Pan, J., D. A. Jay, and P. M. Orton (2007), Analysis of internal solitary waves generated at the Columbia River plume front using SAR imagery, J. Geophys. Res., 112, C07014, doi:10.1029/2006JC003688.
Thorpe, S. A. (2005), The Turbulent Ocean, 439 pp., Cambridge Univ. Press, New York.

Vlasenko, V., N. Stashchuk, and K. Hutter (2005), Baroclinic Tides: Theoretical Modeling and Observational Evidence, 351 pp., Cambridge Univ. Press, New York.

Wang, B., and L. Redekopp (2001), Long internal waves in shear flows: Topographic resonance and wave-induced global instability, Dyn. Atmos. Oceans, 33, 263-302.

Whitham, G. B. (1974), Linear and Nonlinear Waves, Wiley-Interscience, New York.

Zhang, Y., A. M. Baptista, and E. P. Myers (2004), A cross-scale model for $3 \mathrm{D}$ baroclinic circulation in estuary-plume-shelf systems, Cont. Shelf Res., 24, 2187-2214.

N. Stashchuk and V. Vlasenko, School of Earth, Ocean and Environmental Sciences, University of Plymouth, Drake Circus, Plymouth PL4 8AA, UK. (nstashchuk@plymouth.ac.uk; vvlasenko@plymouth.ac.uk) 